\title{
Power Line Cable Transfer Function for the Broadband Power Line Communication Channel
}

\author{
Naing Lin Zaw ${ }^{1}$, Hitke Aung Kyaw ${ }^{2}$, Kyaw Zaw Ye ${ }^{3, *}$ \\ ${ }^{1}$ Department of Automation and Control,National Research University of Electronic Technology (MIET), Bld. 5, Pas. 4806, 124498, \\ Zelenograd, Moscow, Russian Federation \\ ${ }^{2}$ Department of Computer Science,National Research University of Electronic Technology (MIET), Bld. 5, Pas. 4806, 124498, \\ Zelenograd, Moscow, Russian Federation \\ ${ }^{3}$ Software Computer Systems,National Research University of Electronic Technology (MIET), Bld. 5, Pas. 4806, 124498, Zelenograd, \\ Moscow, Russian Federation \\ *Corresponding Author: kyawzawye85@gmail.com
}

Copyright $@ 2013$ Horizon Research publishing all rights reserved.

\begin{abstract}
PLC (Power line communications) use the existing power line infrastructure for communication purposes. While the majority of recent contributions have discussed PLC for high data-rate applications like Internet access or multimedia communication serving a relatively small number of users, in this paper presents a novel approach to model the transfer function of electrical power lines for broadband power line communication. The model has been verified with practical measurements conducted on actual power networks.
\end{abstract}

Keywords Channel Modeling, Communication Channel, Power Line Communications, OFDM, Noise, Modulation, Transfer Function, Two-Port Network

\section{Introduction}

The power line communication (PLC) formally joined (the family of broadband wire) communication systems after NOR.WEB demonstrated the technical feasibility for the transmission of the high-frequency $(>1 \mathrm{MHz})$ signal on the low-voltage (LV) power lines [1]. It is obvious that there are many advantages in using a power line network as a communication channel. The power network is the most pervasive network comparing to any other networks in the world and its availability reaches every sockets in our house. PLC (Power Line Communication) technology takes profits from the advantage of not requiring any additional wiring $[2$, $3]$. On the other hand there are many challenges in using this medium for data communication; all originate from the fact that, this medium was designed for distribution of electrical power, not for communication.

PLC systems fall into two areas: broadband PLC and narrowband PLC. Broadband PLC achieves the characteristics of broadband communication, enabling, for example, fast Internet access or implementation of small
LAN networks. The broadband technology works in frequency range $150 \mathrm{kHz}$ to $34 \mathrm{MHz}$ and its theoretical maximum speed is $200 \mathrm{Mbit} / \mathrm{s}$ [4]. Narrowband PLC is used mainly for specific services including central management of power consumption, tariffing, remote meter reading, commanding, etc. The narrowband technology works up to a maximum frequency of $150 \mathrm{kHz}$ and its theoretical bit rate is of the order of kilobits (up to $2 \mathrm{Mbit} / \mathrm{s}$ ). The possibility of using the power network and systems of narrow-band PLC is the most recent development [5].

A model of the power line channel is required to simulate PLC communications. There are existing two possible methods for modeling the power line channels. The first one applies the methods used for modeling of radio channels. The power line channel is assumed to be a multipath propagation environment. The second alternative applies the methods used to model electricity distribution networks. The chain parameter matrices describing the relation between the input and output voltage and current of a two-port network can be applied to model the transfer function of the communications channel.

\section{PLC Communication Model}

Computer simulation of PLC systems will enable a better understanding of the topic of data transmission over power lines, it will reveal the potentials of these systems, and the area of PLC applicability will be better defined. Sufficiently precise computer models of PLC systems will thus make the process of selecting and deploying new telemetry and management technologies markedly more effective. A model will be described with frequency division of the spectrum using the OFDM technique, where particular carrier frequencies are mapped with 256-state, 64-state or 32-state QAM or QPSK and BPSK modulation [6, 7]. The resulting model is shown in Fig. 1. Model description [8]. A Bernoulli Binary Generator is used as the data source in the proposed model. It is a generator which generates random binary 
numbers according the Bernoulli distribution. In real systems, Bit allocation (mapping) can be obtained from this equation [9].

$$
b_{n}=\log _{2}\left(1+\frac{3}{\left(Q^{-1} \frac{B E R}{4}\right)^{2}} S N R_{n}\right)
$$

Where $\mathrm{b} \mathrm{n}$ is the number of bits at $\mathrm{n}$-sub channel, $S N R_{n}$ is the average signal to noise ratio in partial sub channel $n$ where $Q^{-1}$ is defined as,

$$
Q(x)=\frac{1}{\sqrt{2 \pi}} \int_{x}^{\infty}-\frac{y^{2}}{2} d y .
$$

Channel coding, realized by the Reed-Solomon code $(\operatorname{RS}(15 ; 11))$, was used to ensure data transfer in the communication channel. From the channel coding block, we obtain a serial flow of data. This flow enters the mapping block. The bit sequences are converted to a symbol sequence in the block mapping. The distribution of symbols is the result of mapping. This symbols distribution is dependent on the selected modulation.

The sub-band separation of the useful signal is realized in the Select Rows block. It is necessary to insert the pilot signals and implement an estimate of the transmission channel in the case of coherent system detection. This is realized by the help of the PN Sequence Generator.

Block Complex $(0,0)$ is used to generate the middle carrier frequency. Block Concatenate is used to add up all the carrier frequencies which enter this block. The size of the framework is then adjusted for IFFT in the Pad block and the signal is adjusted for IFFT in the Shift block. After that the signal undergoes the Fast Fourier transform, where data are converted from the frequency domain to the time domain. To avoid inter symbol interference (ISI) the OFDM protective interval is used. It is realized by the Add Cyclic Prefix block. The blocks for calculating, the bit error are used too. The blocks are connected inversely on the receiver side.

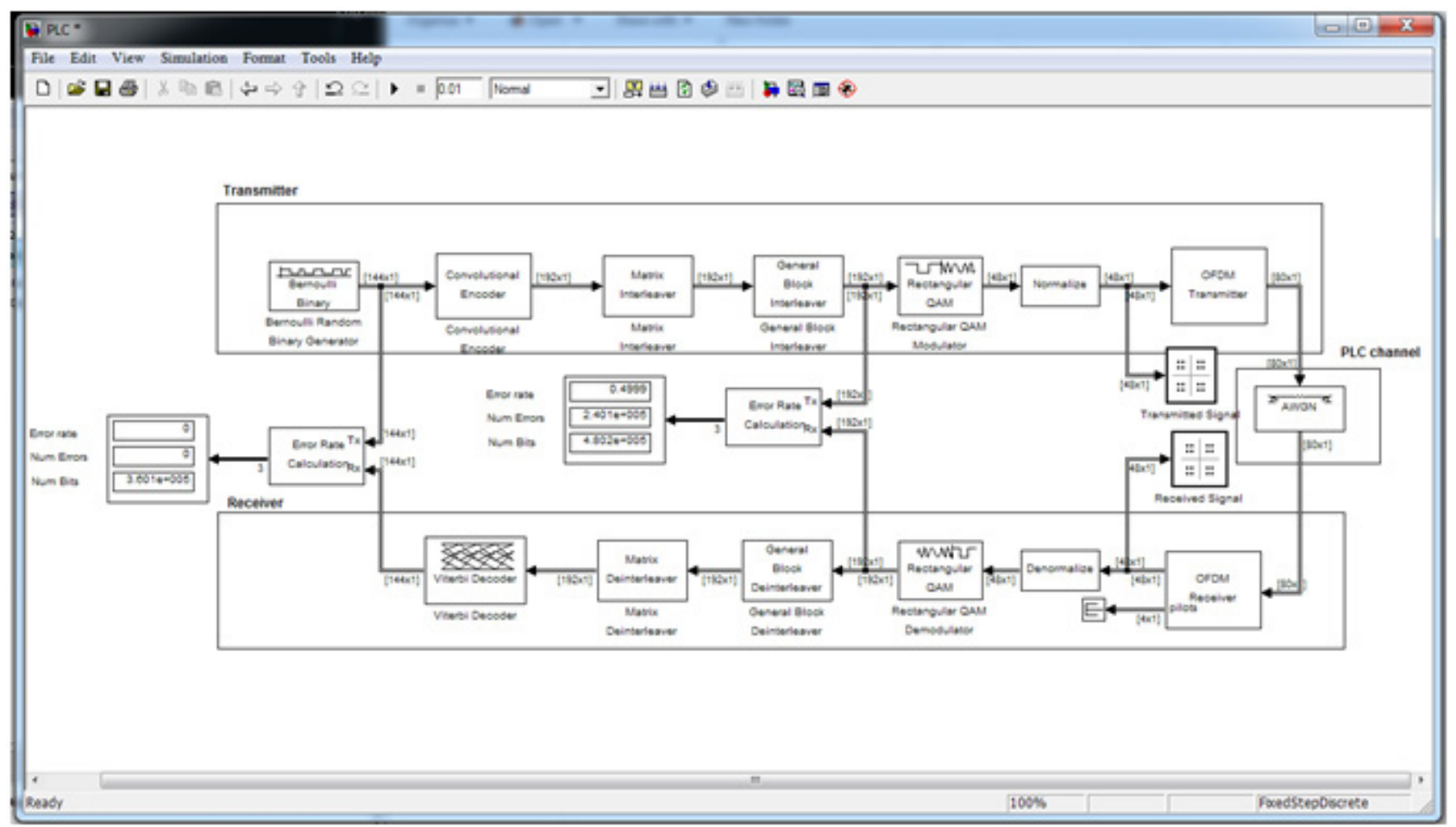

Figure 1. OFDM model

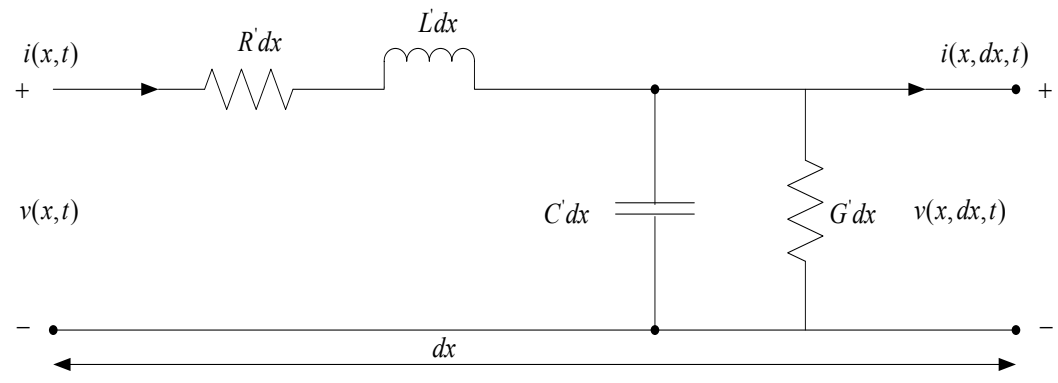

Figure 2. Elementary cell of a transmission line 


\section{Transmission Line Analysis of the Power Line}

The electromagnetic theory states that to achieve efficient point-to-point transmission of power and information, the source energy must be guided. When power lines are used to transmit high frequency communication signals, they can be regarded as transmission lines, which guide the transverse electromagnetic (TEM) waves along them.

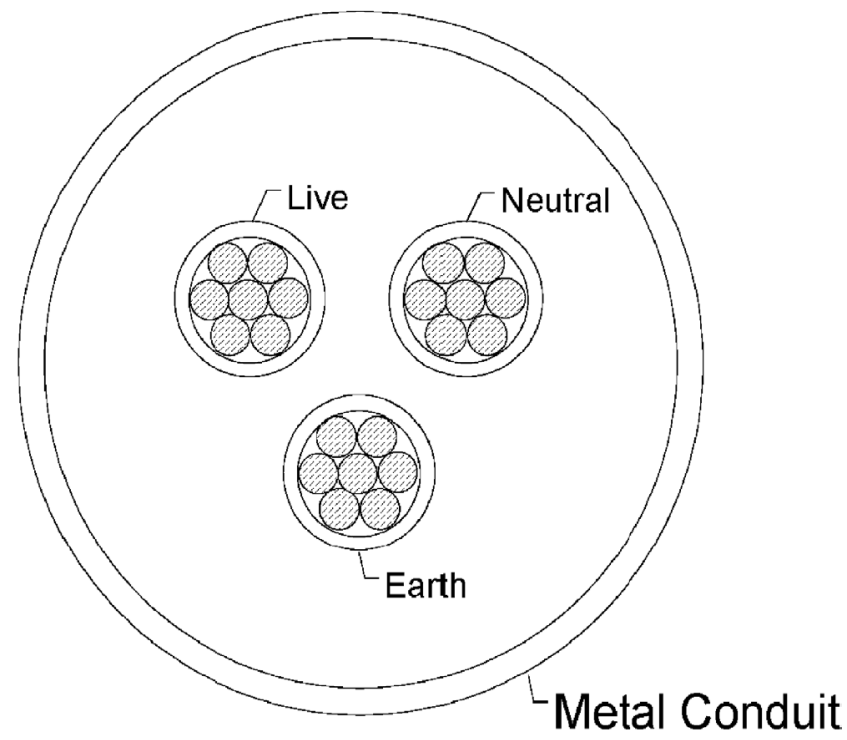

Figure 3. Cross-sectional view of the house service power line.

The cable under study in this paper is the typical single-phase house wirings commonly found in Singapore (as shown in Fig. 3). The cables are made up of stranded copper conductors with PVC insulation. The three cables (live, neutral, and earth) are usually laid inside metal conduits that are embedded inside the concrete wall.

Typically, the live and neutral cables are used as the PLC transmission channel, which can be approximated as a close form of the "two-wire transmission line". According to [11], the two-wire transmission line must be a pair of parallel conducting wires separated by a uniform distance. In the actual installation, the power cables are simply pulled through the conduit and the separation between them is not uniform at all. However, the conduit normally has small cross-sectional area and this limits the variation of the separation between the cables. Hence, the assumption of uniform separation is reasonable in this case.

Based on the above consideration, the paired power cables are regarded as a distributed parameter network, where voltages and currents can vary in magnitude and phase over its length. Hence, it can be described by circuit parameters that are distributed over its length.

In Fig. 4, the quantities $v(z, t)$ and $v(z+\Delta z, t)$ denote the instantaneous voltages at location $z$ and $\Delta z, t$, respectively. Similarly, $i(z, t)$ and $i(z+\Delta z, t)$ denote the instantaneous currents at $z$ and $z+\Delta z$, respectively.
$R$ defines the resistance per unit length for both conductors (in $\Omega / m$ ), $L$ defines the inductance per unit length for both conductors (in $H / m$ ), is the conductance per unit length (in $S / m$ ), and is the capacitance per unit length $(F / m)$.

Based on the lumped-element circuit shown in Fig. 3(b), the two intrinsic line parameters for the transmission line, i.e., the propagation constant and the characteristic impedance $Z_{0}$, can be written as [11]

$$
\begin{gathered}
\gamma=a+j \beta=\sqrt{(R+j \varpi L)(G+j \varpi C)} \\
Z_{0}=\sqrt{\frac{R+j \varpi L}{G+j \varpi C}} .
\end{gathered}
$$

where $\varpi$ is the angular frequency. The real part and the imaginary part $\beta$ of the propagation constant are the attenuation constant (in $N p / m$ ) and phase constant (in $\mathrm{rad} / \mathrm{m}$ ) respectively. Note that both $\gamma$ and $Z_{0}$ are characteristic properties of a transmission line even if the line is infinitely long. In other words, they depend on $R$, $L, G, C$, and $\varpi$, but not the length of the line.

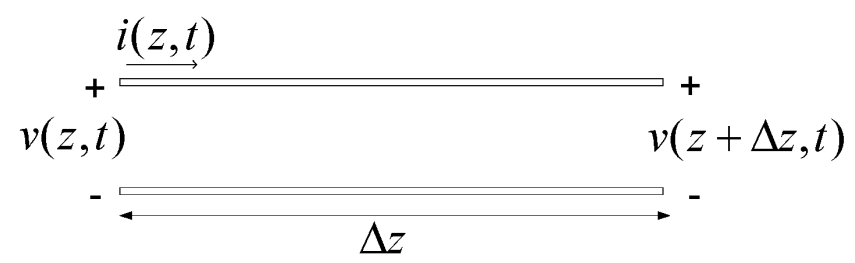

(a)

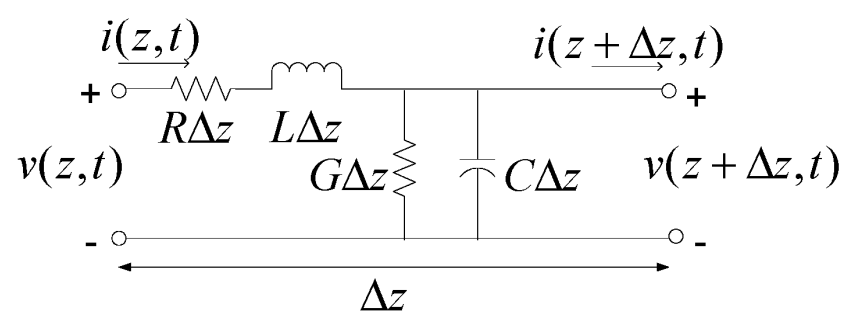

(b)

Figure 4. (a) Voltage and current definitions (b) Equivalent lumped-element circuit of two-wire transmission line

With the power line being modeled as a transmission line, it's $\gamma$ and $Z_{0}$ will dominate the wave behavior along the line. In the model proposed in this paper, they serve as the parameters to model the transfer function of the channel. In the next section, these two parameters are derived for the typical house power cables as shown in Fig. 4.

\section{Primary Parameters of Power Line}

The line can be described by using $\mathrm{R}^{\prime}, \mathrm{L}^{\prime}, \mathrm{C}^{\prime}, \mathrm{G}^{\prime}$ parameters. These parameters denote a resistance, 
inductance, capacity and leakage relative to the length of the line. Let's consider a single-phase signal distribution and management structure which is shown in Fig 5. The wire line includes phase, neutral and ground wires where each wire is inserted into the insulating sleeve and all wires are insulated with own sheath.

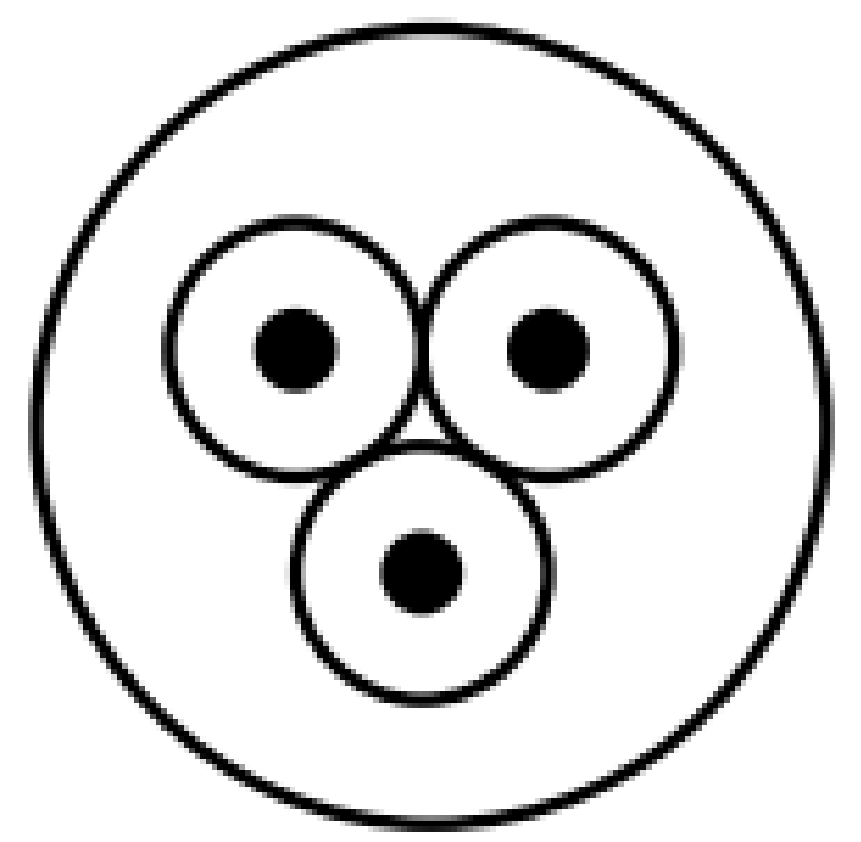

Figure 5. The shape of power line

For the purpose of modeling, we consider the three-phase line as the two conductors and one transmission conductor, which they are a conductive core and they are surrounded by the same dielectric material. Then it is possible to determine the primary parameters with the equations [11]:

$$
\begin{gathered}
R^{\prime}=\sqrt{\frac{\mu_{r} \mu_{0} f}{\pi \sigma a^{2}}}\left[\frac{\frac{d}{2 a}}{\sqrt{\left(\frac{d}{2 a}\right)^{2}-1}}\right] \\
L^{\prime}=\frac{\mu_{r} \mu_{0}}{\pi} \cosh ^{-1}\left(\frac{d}{2 a}\right) \\
C^{\prime}=\frac{\pi \varepsilon_{r} \varepsilon_{0}}{\cosh ^{-1}\left(\frac{d}{2 a}\right)} \\
G^{\prime}=2 \pi f C \tan \delta
\end{gathered}
$$

Where $d$ is a distance between the centre of conductors, $a$ is a radius of conductor, $\sigma$ is a copper conductivity, $\varepsilon_{r}$ indicates a relative permittivity of insulation, $\varepsilon_{0}$ is a permittivity of vacuum, $\tan \delta$ is a factor variance a $\mu_{r}$ is a relative magnetic permittivity of the copper.

\section{The Sources of Interference}

Besides simulation of transfer characteristic, it is necessary to identify possible sources of interference because the power line has a significant attenuation of signal and various interference and noise. Therefore the data transfer has a high error rate without any checking algorithm.

The fundamental influence on data transmission over power lines are mainly the negative characteristics of power networks. Can be summarized in a few points:

- Mismatched impedance

- Attenuation on the communication channel

- Interference (noise)

- Interference changing in time

Fig. 6 shows a simplified block model of the PLC communication channel, in which the described characteristics and parameters are included. The parameters of interference, except noise, are represented as a time variable linear filter described by the frequency parameters. Noise is depicted as additive random interference process.

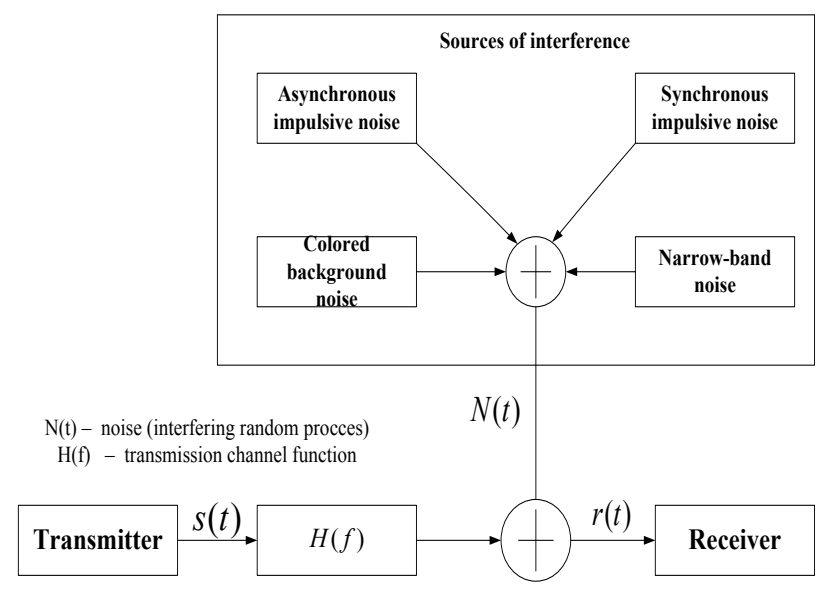

Figure 6. PLC channel model

This model captures the whole range of parameters which are necessary for a model of the communication system with corresponding characteristics, although this model is schematically simplified in the figure.

Transmission function and noise can be either estimated from the measurement or derived from the theoretical analysis.

In this paper, we deal with four different types of noise:

Background noise: it is every time present in the network. It is caused by assembling of multiple sources of noise with low power. It can be described by a PSD (Power Spectral Density) that it declines with a growly frequency. The background power noise density can be described with equation:

$$
A(f)=A_{\infty}+A_{0} \cdot e^{\frac{-f}{f_{0}}}
$$

where $A \infty$ is power density for $\mathrm{f} \rightarrow \infty$, and A 0 is a differences between $\mathrm{A}(\infty)$ and $\mathrm{A}(0)$. This model enables modeling background noise as a white noise process, which gets a spectral coloring by a filter. 
Narrow-band noise: this noise primary originates from the broadcasting stations that they transmit in a long, middle a short wave range. The amplitude can be changed in dependence on time and place. The narrow-band noise can be modeled as a sum of multiple sine noise with different amplitude:

$$
n(t)=\sum_{i=1}^{N} A_{i}(t) \cdot \sin \left(2 \pi f_{i} t+\varphi_{i}\right)
$$

where $n$ is a number of waves of differencing frequencies $f_{i}$, amplitude $A_{i}(t)$ and phase $\varphi_{i}$. The amplitude $A_{i}$ $(t)$ is a constant in simplest case but it can be established from broadcast transmission. The phase $\varphi_{i}$ is randomly established from interval $[0 ; 2 \pi]$.

Asynchronous impulsive noise: this type of noise is characterized by high and short spikes of voltage with length $10-100 \mu \mathrm{s}$. These spikes can reach up to $2 \mathrm{kV}$ level. This noise is the cause of the switching equipments in the distribution network. These kinds of noise are considered as a part of background noise.

Synchronous impulsive noise: is caused by thrusters in light dimmers and copiers. They are bursts of interference spikes with repeating of period. Synchronous impulse noise can be modeled by a source of white noise with a spectral coloring together with a periodical switching of rectangular wrap (Fig. 7).

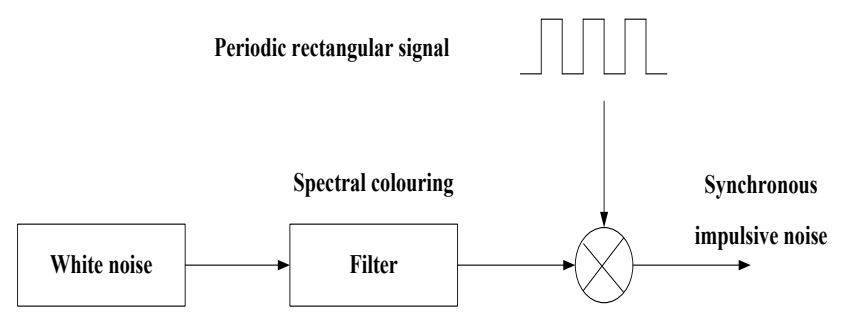

Figure 7. Synchronous impulsive noise

\section{Transfer Function Modeling}

With $\gamma$ and $Z_{0}$, the transfer function of the PLC channel can be determined. The different mechanisms by which the PLC signal is attenuated need to be understood. There are three main types of attenuation for a wave propagating in the forward direction. The first one is the line attenuation, which is caused by the heat loss and radiations along the power line. This line attenuation is always present and it depends on the length of the wave path and the frequency of the wave. The second type of attenuation is caused by reflections arising from the points of impedance discontinuities on the propagation channel. The reflected wave from the unmatched points will interfere with the original incident wave. This kind of interferences may be constructive or destructive, giving rise to attenuation if it is destructive. The last type of attenuation is caused by the delayed version of the forward propagating wave falling out of phase with the main incident forward wave, giving rise to destructive interference and hence overall signal attenuation.

As a result, frequency-domain modeling approach is suggested because it is very hard to use the time-domain modeling approach to account for all these reflected and delayed paths in the power network. Since most in-house power line network installations in Singapore are radial, the PLC channel can be regarded as a -branch network as shown in Fig. 8 below. Obtaining the scattering matrix of such a network is quite complex and, the channel is divided into a group of cascaded single-branch networks. The scattering matrix for each single-branch network is derived in the following section and the scattering matrix of the whole channel can then be determined by using the chain-scattering matrix method.

To analyze the S-parameters of a single-branch network, the following diagram shown in Fig. 8 is used. In this derivation, the power line on the direct signal path (excluding the branches) is defined as the path line. Let $l_{1}$ be the cable length from left end of the path line to the tap point, $l_{2}$ the cable length of the cable branched off from the tap point and the cable length from the tap point to the right end of the path line. Using transmission line theory, $Z_{i n 1}$, $Z_{\text {in } 2}, Z_{\text {in }}, \Gamma_{1}$, and $\Gamma_{2}$ can be determined

$$
\begin{gathered}
Z_{i n 1}=Z_{0} \cdot \frac{Z_{L}+Z_{0} \cdot \tanh \left(\gamma \cdot l_{3}\right)}{Z_{0}+Z_{l} \cdot \tanh \left(\gamma \cdot l_{3}\right)}(\Omega) \\
Z_{\text {in } 2}=Z_{0} \cdot \frac{Z_{b}+Z_{0} \cdot \tanh \left(\gamma \cdot l_{2}\right)}{Z_{0}+Z_{b} \cdot \tanh \left(\gamma \cdot l_{2}\right)}(\Omega) \\
Z_{\text {in }}=Z_{0} \cdot \frac{\left(Z_{i n 1} / / Z_{i n 2}\right)+Z_{0} \cdot \tanh \left(\gamma \cdot l_{1}\right)}{Z_{0}+\left(Z_{i n 1} / / Z_{i n 2}\right) \cdot \tanh \left(\gamma \cdot l_{1}\right)}(\Omega)
\end{gathered}
$$

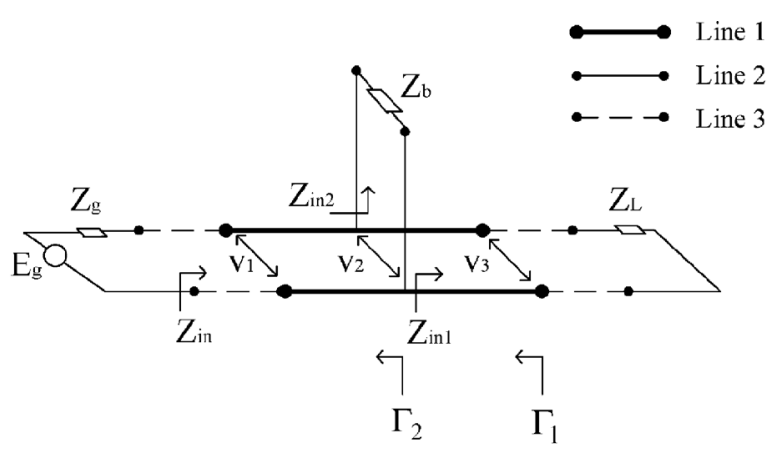

$Z_{g}: \quad$ Source impedance $(50 \Omega)$

$Z_{l}: \quad$ Load impedance at path end $(50 \Omega)$

$Z_{b}$ : Load impedance at branch end

$Z_{\text {in }}$ : Input impedance of the network on the right of the tap

$Z_{\text {in } 2}$ : Input impedance of the branch network

$Z_{i n}: \quad$ Input impedance of the single-branch network

$\Gamma_{1}$ : Reflection coefficient from path end

$\Gamma_{2}$ : Reflection coefficient from the tap point

Line1: The path power line with line parameter $\left(Z_{0}, \gamma\right)$

Line2: The branch power line with line parameter $\left(Z_{0}{ }^{\prime}, \gamma^{\prime}\right)$

Line3: The transmission line with $50 \Omega$ characteristic impedance (negligible length)

Figure 8. Detailed diagram for single-branch network. 


$$
\begin{gathered}
\Gamma_{1}=\frac{Z_{L}-Z_{0}}{Z_{L}+Z_{0}} \\
\Gamma_{2}=\frac{\left(Z_{i n 1} / / Z_{i n 2}\right)-Z_{0}}{\left(Z_{i n 1} / / Z_{i n 2}\right)+Z_{0}}
\end{gathered}
$$

According to [12], the $S_{11}$ and $S_{21}$ of the network scattering matrix is given by

$$
\begin{gathered}
S_{11}=\frac{Z_{\text {in }}-50}{Z_{\text {in }}+50} \\
S_{21}=2 \cdot \frac{V_{3}}{E_{9}} .
\end{gathered}
$$

Since it is not easy to obtain the ratio of $E_{9}$ and $V_{3}$ in (17), $S_{21}$ can be computed indirectly by

$$
S_{21}=2 \cdot \frac{V_{3}}{V_{2}} \cdot \frac{V_{2}}{V_{1}} \cdot \frac{V_{1}}{E_{9}} .
$$

Where

$$
\frac{V_{1}}{E_{9}}=\frac{Z_{\text {in }}}{Z_{\text {in }}+Z_{9}}
$$

By applying shifting in the reference planes [12], i.e,

$$
\begin{gathered}
\frac{V_{3}}{V_{2}}=\frac{\left(1+\Gamma_{1}\right) \cdot e^{-\gamma \cdot l_{3}}}{1+\Gamma_{1} \cdot e^{-\gamma \cdot l_{3}}} \\
\frac{V_{2}}{V_{1}}=\frac{\left(1+\Gamma_{2}\right) \cdot e^{-\gamma \cdot l_{1}}}{1+\Gamma_{2} \cdot e^{-\gamma \cdot l_{1}}}
\end{gathered}
$$

and then substitute (19), (20), and (21) into (18), $S_{21}$ can be obtained. Similar method can be used to determine $S_{12}$ and

\section{$S_{22}$ by simply swapping the source and load locations.}

After analyzing the single-branch network, the remaining task is the determination of the scattering matrix for a cascade of several single-branch networks. Using the microwave theory, there are generally two methods that can be used. The first method is to use the chain scattering matrix (or T-matrix), and the second is to use signal flow graph. For the sake of easier computation, the first method is used in this paper.

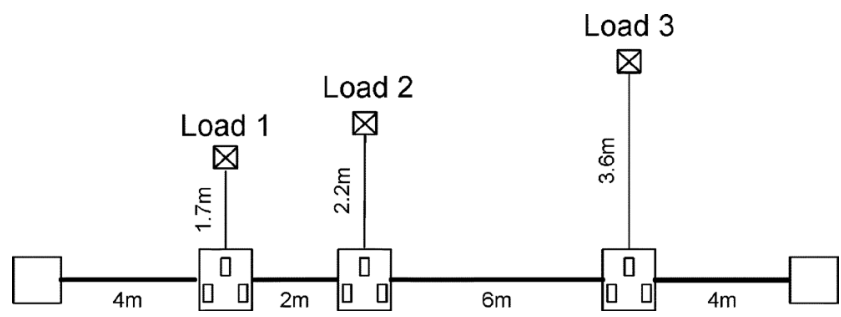

Figure 9. Configuration of simulated network.
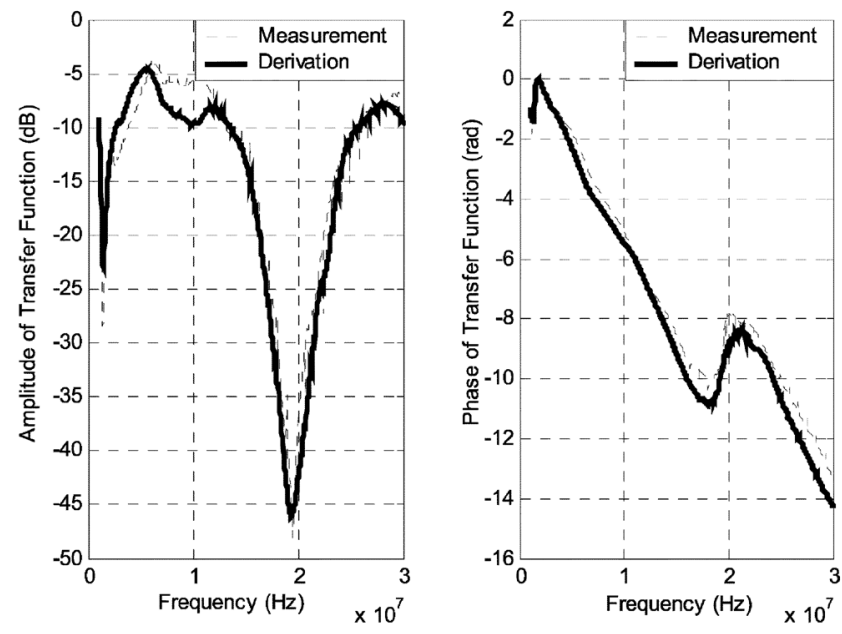

Figure 10. Comparison of derived transfer function with measurement for the 3-branch network

In retrospect, the T-matrix for the whole network can be obtained by the product of all the T-matrices of the cascaded portions. The relation between the $\mathrm{S}$-matrix and T-matrix is shown as follows $[13,14]$ :

$$
[T]=\left(\begin{array}{ll}
T_{11} & T_{12} \\
T_{21} & T_{22}
\end{array}\right)=\left(\begin{array}{cc}
\frac{1}{S_{12}} & -\frac{S_{22}}{S_{21}} \\
\frac{S_{11}}{S_{21}} & S_{12}-\frac{S_{11} S_{22}}{S_{21}}
\end{array}\right)
$$

So the total T-matrix for the N-branch network will be

$$
[T]=\prod_{k=1}^{N}\left[T_{k}\right],
$$

where $T_{k}$ is the T-matrix for the $k$ - th cascaded portion in the network. Finally, the S-matrix for the whole network can be obtained by using the following conversion equation:

$$
[S]=\left(\begin{array}{ll}
S_{11} & S_{12} \\
S_{21} & S_{22}
\end{array}\right)=\left(\begin{array}{cc}
\frac{T_{21}}{T_{11}} & T_{22}-\frac{T_{21} T_{12}}{T_{11}} \\
\frac{1}{T_{11}} & -\frac{T_{12}}{T_{11}}
\end{array}\right)
$$

The $S_{21}$ term in (24) gives the network transfer function.

To verify the above derivations, the transfer function for a network with three branches as shown in Fig. 9 is measured. Loads 1, 2, and 3 in the network under test are light dimmer, $\mathrm{TV}$, and electric fan, which are typical household appliances. All the loads are in operating condition. The measured impedances of these electrical appliances over $1 \sim 30 \mathrm{MHz}$ are given in the Appendix.

In Fig. 10, the amplitude and the phase angle of the derived transfer functions for this 3-branch network are compared with those obtained from measurements. It can be seen that the derived and measured transfer functions match each other closely, demonstrating that the model can accurately predict the channel transfer function of the PLC medium including the positions of attenuation notches in the 
frequency domain. The strong attenuation notch at about $20 \mathrm{MHz}$ is mainly caused by the impedances of the branched loads. It can be observed in Fig. 13 that all the three loads have very small impedances at frequency around $20 \mathrm{MHz}$. As a result, the majority of the signals in this frequency range will be shorted out when they travel along the channel.
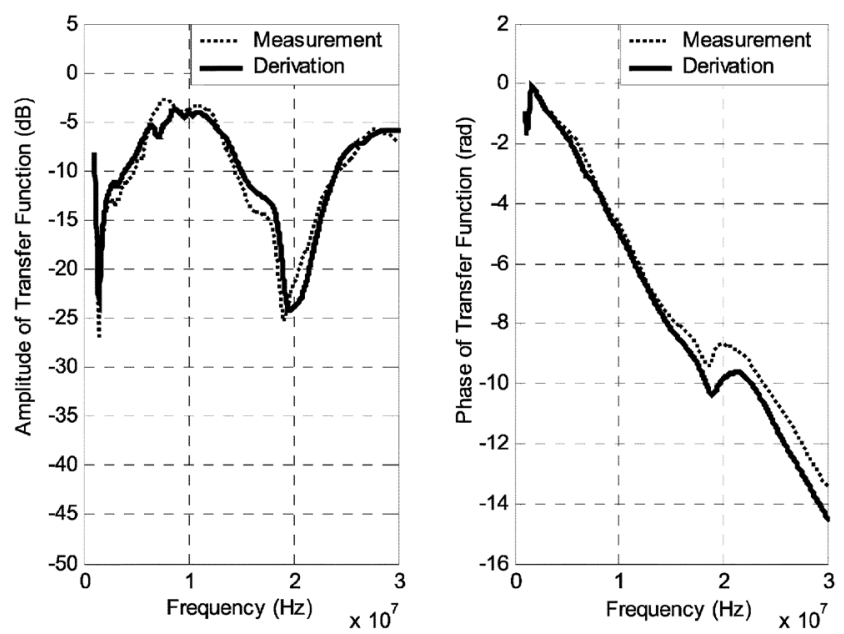

Figure 11. Comparison of derived transfer functions with measurement after the change of the load condition.

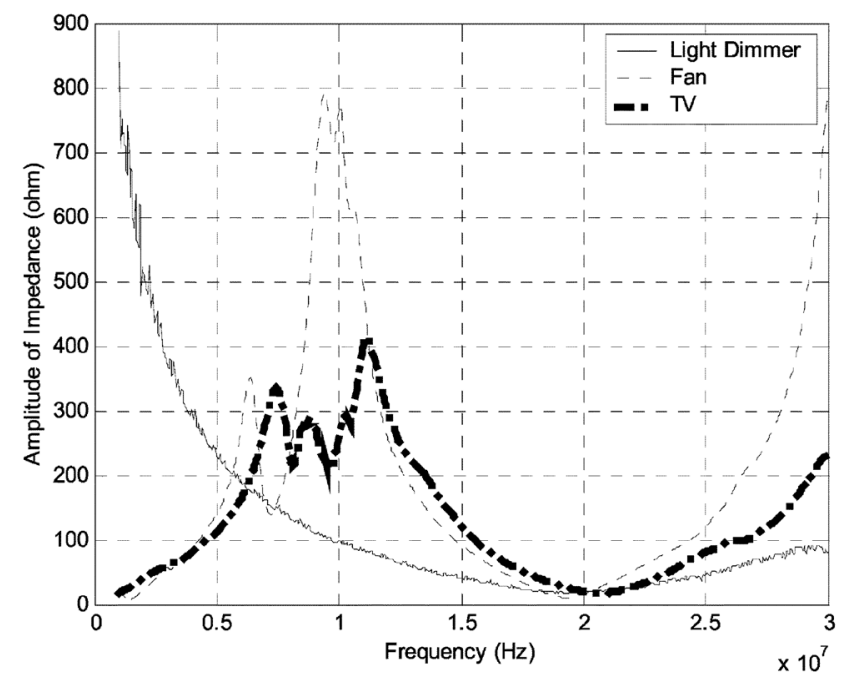

Figure 12. Impedances of some typical appliances in operating condition.

To demonstrate the flexibility and versatility of this model, Load 1 (light dimmer) is unplugged from the network. The newly derived and measured transfer functions are then compared as shown in Fig. 11. The strength of the attenuation notch near $20 \mathrm{MHz}$ has reduced to about $25 \mathrm{~dB}$. Once again, both the measurement and derivation results match closely to each other, verifying the capability of this model in predicting the behavior of the power line channel.

Knowing the characteristics of the power line channel will be of great help in analyzing the performance of different communication schemes as well as identifying potential difficulties.

This modeling approach can be used to model power networks in different configurations, as long as the construction and dimension of the power cables are known.

\section{Conclusion}

In this paper, the LV single-phase power line is modeled as a transmission line to compute the two intrinsic line parameters namely, the characteristic impedance and the propagation constant. The model takes into consideration of the type of cable used and the cable mounting method. Making use of these intrinsic parameters as the model parameters, the LV power network is then regarded as an $\mathrm{N}$-branch network, which is subdivided into several cascades of smaller networks. The channel transfer function is later determined by combining the scattering matrices of the cascaded sub networks. Both of the model parameters and the transfer characteristics have been successfully verified through practical measurements on actual power line.

\section{REFERENCES}

[1] P. A. Brown, "High frequency conditioned power networks," in UTC Annu. Conf. Proc., July/Aug. 1995.

[2] Murzin, M., Y. Volkov , ELECTRICAL ENGINEERING, textbook for undergraduate students in areas of training graduate "Computer Science", "Electronics and Microelectronics", "Design and technology of electronic media" / Moscow, 2007. Ser. Textbook

[3] H. Hrasnica, A. Haidine, R. Lehnert, “ Broadband Powerline Communications Network Design, "Willey , c2004. 275 s. ISBN 0-470-85741-2.

[4] ORGO N, M.: PLC/BPL and Next Generation Networks, In: POWER-COM - Conference Communication over MV and LV power lines, Praha, 2007.

[5] MLYNEK, P.-MISUREC, J.-KOUTNY, M. The Communication Unit for Remote Data Acquisition via the Internet : In Proceedings of the 7th WSEAS International Conference on Circuits, Systems, Electronics, Control and Signal Processing (CSES'08), Puerto de La Cruz, Spain, WSEAS Press, 2008, pp. 168-173.

[6] MISUREC, J.: The Data Acquisition via PLC in Energetics, In 32nd International Conference on Telecommunications and Signal Processing - TSP'2009, pp. 1-4.

[7] KRAJSA, O.-SILHAVY, P.-KOUTNY, M. : Half-Over-lapped Filtered MultiTone Modulation for PowerLine Communication Systems, In Proceedings of the 13th WSEAS International Conference on Systems, Rhodos, WSEAS Press, 2009, pp. 596-599.

[8] KRAJSA, O.-SILHAVY, P. :Half-Overlapped Filtered MultiTone Modulation, its Implementation and Comparison with Non-Overlapped Filtered MultiTone Modulation, In Proceedengs of The 7th WSEAS International Conference on Circuits, Systems, Electronics, Control \& Signal Processing, 1, Puerto De La Cruz, Spain, WSEAS, 2008, pp. 272-27.

[9] KOUTNY, M.-KRAJSA, O.-MLYNEK, P. : Modelling of 
PLC Communication for Supply Networks, In Proceedings of the 13th WSEAS International Conference on Communication, Rhodos, WSEAS Press, 2009, pp. 185-189.

[10] Jiří VODRÁŽKA, Multi-Carrier Modulation and MIMO Principle Application on Subscriber Lines RADIOENGINEERING, VOL. 16, NO. 4, DECEMBER 2007.

[11] D. K. Cheng, Fundamental of Engineering Electromagnetics.
Reading, MA: Addison-Wesley. 2010.

[12] M. Babic, M. Hagenau, K. Dostert, J. Bausch, “ Theoretical postulation of PLC channel mode, " Open PLC European Research Alliance (OPERA). 2005.

[13] D. M. Pozar, Microwave Engineering. New York: Wiley. 2011.

[14] G. Gonzalez, Microwave Transistor Amplifiers analysis and design (2nd edition). Guillermo Gonzalez. 2012. 